Recepción: 20 / 04 / 2017

Aceptación: 20 / 05 / 2017

Publicación: 15 / 05 / 2017

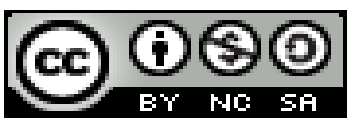

Ciencias de la Salud

Artículo de Investigación

\title{
Caracterización clínicoepidemiológica de pacientes especiales con maloclusion
}

\section{Clinical epidemiological characterization}

of special patients with malocclusion

\section{Caracterização clínico-epidemiológica de pacientes especiais com má oclusão}

Juan Oliveira del Rio ${ }^{\mathrm{I}}$ juanoliveiradelrio@hotmail.com

Sandra Sandoval Pedauga II sandra.sandoval@hotmail.com

Freya Andrade Vera ${ }^{\text {III }}$ freya.andrade@ hotmail.com

Correspondencia: juanoliveiradelrio@ hotmail.com

\footnotetext{
${ }^{\text {I } O d o n t o l o g o, ~ U n i v e r s i d a d ~ d e l ~ Z u l i a, ~ M a r a c a i b o, ~ V e n e z u e l a . ~}$

${ }^{\text {II }}$ Odontologa, Universidad del Zulia, Maracaibo, Venezuela.

III Odontologa, Universidad Católica de Santiago De Guayaquil, Guayaquil. Ecuador.
} 


\section{Resumen}

Se realizó un estudio descriptivo y transversal de 76 niños especiales de 5-19 años con maloclusiones, quienes acudieron a consulta en el período comprendido de enero a junio del 2016, con vistas a caracterizarles según variables clínicas y epidemiológicas de interés : edad, sexo, principales hábitos, factores de riesgos y características clínicas; las que fueron relacionadas y analizadas estadísticamente. Entre los resultados sobresalientes figuraron el sexo masculino y los pacientes especiales con retraso leve, la edad de 15-19 años. La clasificación clase I de Angle fue la más observada en los retrasados mentales leves, siendo las restauraciones deficientes el factor de riesgo que prevaleció. La característica clínica preponderante en ambos sexos fue la versión vestibular de los incisivos superiores.

Palabras claves: Pacientes especial; maloclusion; factores de riesgo. 


\begin{abstract}
A descriptive and cross - sectional study of 76 special children aged 5-19 years with malocclusions was carried out, who went to the clinic from January to June of 2016 to characterize them according to clinical and epidemiological variables of interest: age, sex, main habits, risk factors and clinical characteristics; which were statistically related and analyzed. Outstanding results included males and special patients with mild delay, age 15-19 years. Angle class I classification was the most observed in mild mental retardation, with poor restorations being the risk factor that prevailed. The predominant clinical feature in both sexes was the vestibular version of the upper incisors.
\end{abstract}

Key words: Patients special; maloclusion; risk factor's. 


\section{Introducción.}

El paciente discapacitado o especial es aquel niño, joven o adulto que presenta signos y síntomas que lo alejan de la normalidad, que puede encontrarse médicamente comprometido, o con algún tipo de discapacidad física, psíquica, sensorial o problemas del comportamiento. ${ }^{1}$ En estomatología el paciente especial es aquella persona que para su atención exige maniobras, conceptos, equipamiento y personas de ayuda especiales, o sea, escapan a los programas y rutinas estándar que se llevan a cabo en el mantenimiento de la salud bucodental de la población. ${ }^{1}$

Diferentes factores prenatales, perinatales y posnatales pueden generar discapacidad. Los factores prenatales como afecciones hereditarias dominantes y recesivas, aberraciones cromosómicas, infecciones (rubiola y toxoplasmosis), intoxicaciones con alcohol y drogas durante el embarazo, radiaciones, desnutrición etc., predominan en un $90 \%$. Los perinatales como traumas, falta de oxígeno durante el nacimiento, o prematurez extrema, comprenden un $5 \%$, y posnatales como infecciones (meningitis y encefalitis), intoxicación, trauma y tumoraciones, comprenden el otro $5 \% .^{2}$

Las Naciones Unidas y la Organización Mundial de la Salud coinciden en que las estadísticas demuestran que el $10 \%$ de la población mundial es portadora de discapacidades de distintos tipos y gravedades. ${ }^{3}$

Existe un aumento del por ciento de discapacitados a nivel mundial, sobre todo en las edades de 6 y 16 años, más frecuente en niños que en niñas. ${ }^{2}$ Este incremento puede deberse a que estos niños tienen hoy una esperanza de vida más larga gracias a los progresos de la medicina, o al hecho de que cada vez hay más jóvenes discapacitados como consecuencia de accidentes o de actos 
violentos. También parece que tiende a aumentar el número de discapacitados entre las personas de edad. $^{1}$

En el Ecuador, el Gobierno de la Revolución Ciudadana ha asumido el reto de construir una "Patria para todos y todas", una sociedad más justa y solidaria que promueva la inclusión social de todos los sectores, especialmente de aquellos grupos que requieren atención prioritaria como es el caso de las personas con discapacidad. En nuestro país, de acuerdo al VI Censo de Población y V de Vivienda (INEC, 2001), el porcentaje de la población ecuatoriana que señala tener discapacidad fue de $4.7 \%$, datos que en el transcurso de una década ascendió a 5.6\%, según los datos del VII Censo de Población y VI Vivienda (INEC, 2010). Por otro lado, el estudio "Ecuador: la discapacidad en cifras" (CONADIS - INEC, 2005), establece que el 12.14\% de la población ecuatoriana tiene algún tipo de discapacidad; y, el estudio bio-psico-social Misión Solidaria Manuela Espejo, da cuenta de 294.803 personas con discapacidad. ${ }^{4}$

La mayoría de los autores ${ }^{1,5,6-7}$ plantean que en las personas con discapacidad mental se presentan numerosos problemas de salud bucal, provocados por varios factores de riesgo, como deficiente higiene bucal, consumo de dieta cariogénica rica en azúcares, inducida por los padres, dieta blanda, dificultades para el tratamiento y pobre educación higiénico sanitaria. La proporción de la caries dental en ellos es superior que en los pacientes normales, el estado periodontal pobre y los problemas bucales más severos por las anomalías dentales que presentan desde edades muy tempranas.

La alta prevalencia de maloclusiones como, mordida cruzada posterior, clase III por insuficiente desarrollo del maxilar superior y protrusión mandibular, el $45 \%$ presenta clase I. En los Down la incidencia de caries es baja con respecto a otros grupos de deficientes psíquicos. Existe alta 
susceptibilidad a la enfermedad periodontal y baja predisposición a contraer caries. Algunos de ellos padecen cardiopatías congénitas con afección valvular por lo que es necesaria la prevención de la endocarditis bacteriana a la hora de su atención. ${ }^{6}$

Los estudios coinciden en que en el tratamiento odontológico al paciente con retraso mental la prevención es la consideración más importante, que debe iniciarse desde edades tempranas. La atención debe ser sistemática y periódica, con aplicación de técnicas de manejo adecuado, imprescindible para lograr resultados satisfactorios. En la educación y desarrollo de estos niños el papel decisivo corresponde a los padres y familia, auxiliados por los educadores y el equipo de salud. ${ }^{1}$

A nivel mundial la mayoría de los discapacitados no reciben tratamientos odontológicos acorde a sus necesidades. El odontólogo necesita más familiarización, profundización y capacitación sobre este tipo de paciente, para brindar una atención odontológica con calidad. ${ }^{1}$

\section{Metodología.}

Se realizo un estudio descriptivo transversal que acudieron a consulta con la finalidad de caracterizar los pacientes especiales con maloclusiones según variables clínicas y epidemiológicas.

La población de estudio estuvo conformada por 76 pacientes especiales cuyas edades fueron entre 5 y 19 años de ambos sexos

Pacientes especiales: Es aquel niño, joven o adulto que presenta signos y síntomas que lo alejan de la normalidad, que puede encontrarse medicamente comprometido, o con algún tipo de discapacidad física, psíquica , sensorial o problema de comportamiento. ${ }^{8}$ 
Se consideraron aquellos pacientes:

Retrasados mentales (leve y moderado) según diagnostico previamente establecido.

Variables utilizadas fueron: edad, sexo, hábitos deformantes, factores de riesgo.

Igualmente, entre las características clínicas figuraron:

- Versión vestibular de los incisivos superiores: protusión dentoalveolar superior.

- Adaquia o mordida abierta anterior: cuando había separación de las arcadas superior e inferior en el sector anterior.

- Retrognatismo mandibular: si existía un perfil convexo (perfil de pájaro) con resalte exagerado.

- Hipotonía del labio superior: cierre bilabial insuficiente por el labio superior corto.

La recogida del dato primario se realizo a través de una planilla confeccionada por el autor.

Los datos obtenidos fueron procesados utilizando el paquete estadístico SPSS versión 5.0 y los resultaos obtenidos fueron plasmado en tablas simples y de doble entrada. Se utilizo como medida de resumen las frecuencias absolutas y elativas en porcientos. 


\section{Resultados.}

Tabla 1 Pacientes especiales con maloclusiones según edad y sexo.

\begin{tabular}{|l|c|c|c|c|c|c|}
\hline \multirow{2}{*}{$\begin{array}{l}\text { Grupos de } \\
\text { edades }\end{array}$} & \multicolumn{4}{|c|}{ Sexo } & \multicolumn{2}{c|}{ Total } \\
\cline { 2 - 7 } & \multicolumn{2}{|c|}{ Femenino } & \multicolumn{2}{c|}{ Masculino } & No & $\%$ \\
\hline $5-9$ & 9 & 64.2 & 5 & 35.8 & 14 & 18.4 \\
\hline $10-14$ & 13 & 68.4 & 6 & 31.6 & 19 & 25.0 \\
\hline $15-19$ & 5 & 11.6 & 38 & 88.4 & 43 & 56.6 \\
\hline Total & 27 & 35.5 & 49 & 64.5 & 76 & 100.0 \\
\hline
\end{tabular}

En la tabla 1 se muestra la distribución de la población por grupos de edades y sexo donde se evidencia que de un total de 76 pacientes examinados predomino el sexo masculino con $64.5 \%$.

El grupo de edad que predomino fue el de 15 a 19 años con 43 pacientes que represento el $56.6 \%$ del total.

Tabla 2 Pacientes especiales con maloclusiones según tipo de retraso y sexo.

\begin{tabular}{|l|c|c|c|c|c|c|}
\hline \multirow{2}{*}{$\begin{array}{l}\text { Tipo de } \\
\text { retraso }\end{array}$} & \multicolumn{4}{|c|}{ Sexo } & \multicolumn{2}{c|}{ Total } \\
\cline { 2 - 7 } & \multicolumn{2}{|c|}{ Femenino } & \multicolumn{2}{c|}{ Masculino } & No & $\%$ \\
\hline Leve & 14 & 31.5 & 32 & 69.5 & 46 & 60.5 \\
\hline Moderado & 13 & 43.4 & 17 & 56.6 & 30 & 39.5 \\
\hline Total & 27 & 35.6 & 49 & 64.4 & 76 & 100.0 \\
\hline
\end{tabular}

En la tabal 2 se observa un mayor por ciento de pacientes diagnosticado con retraso leve (60.5\%), prevaleciendo el sexo masculino. 
Tabla 3 Pacientes especiales según hábitos deformantes

\begin{tabular}{|c|c|c|c|c|}
\hline \multirow{2}{*}{$\begin{array}{c}\text { Hábitos } \\
\text { deformantes }\end{array}$} & \multicolumn{4}{|c|}{ Sexo } \\
\cline { 2 - 5 } & \multicolumn{2}{|c|}{ Femenino } & \multicolumn{2}{c|}{ Masculino } \\
\hline $\begin{array}{c}\text { No } \\
\text { digital }\end{array}$ & 7 & 25.9 & 8 & 16.3 \\
\hline $\begin{array}{c}\text { Succión de } \\
\text { chupete }\end{array}$ & 0 & 0 & 0 & 0 \\
\hline Queilofagia & 8 & 29.6 & 0 & 0 \\
\hline Onicofagia & 17 & 62.9 & 7 & 14.2 \\
\hline
\end{tabular}

Véase en la tabla 3 la distribución de la población en estudio según hábitos deformantes y sexo donde se evidencia un mayor por ciento en el sexo femenino y el habito deformante que predomino fue la onicofagia.

Tabla 4 Pacientes especiales según defunciones neuromusculares

\begin{tabular}{|l|c|c|c|c|}
\hline \multirow{2}{*}{$\begin{array}{l}\text { Disfunciones } \\
\text { neuromusculares }\end{array}$} & \multicolumn{4}{|c|}{ Sexo } \\
\cline { 2 - 5 } & \multicolumn{2}{|c|}{ Femenino } & \multicolumn{2}{c|}{ Masculino } \\
\hline No & \multicolumn{2}{|c|}{$\%$} & No & $\%$ \\
\hline Disfunción lingual & 14 & 51.8 & 14 & 28.5 \\
\hline Respiración bucal & 8 & 29.6 & 9 & 18.3 \\
\hline Disfuncion labial & 6 & 22.2 & 2 & 22.2 \\
\hline
\end{tabular}

Como se muestra en la tabla 4 la distribución de la población en estudio según disfunciones neuromusculares y sexo se evidencia un mayor por ciento en el sexo femenino (disfunción lingual $51.8 \%)$ 
Tabla 5 Pacientes especiales según factores de riesgos.

\begin{tabular}{|l|c|c|c|c|}
\hline \multirow{2}{*}{ Factores de riesgos } & \multicolumn{4}{|c|}{ Sexo } \\
\cline { 2 - 5 } & \multicolumn{2}{|c|}{ Femenino } & \multicolumn{2}{c|}{ Masculino } \\
\hline No & 1 & 3.7 & 3 & 6.1 \\
\hline $\begin{array}{l}\text { Perdida prematura } \\
\text { dientes } \\
\text { permanentes }\end{array}$ & 0 & & 2 & 4.0 \\
\hline $\begin{array}{l}\text { Perdida prematura } \\
\text { de dientes } \\
\text { temporales }\end{array}$ & 14 & 51.8 & 25 & 51.0 \\
\hline Caries proximales & 33 & 122.0 & 36 & 73.4 \\
\hline $\begin{array}{l}\text { Restauaciones } \\
\text { defiecientes }\end{array}$ & 1 & & 3 & 6.1 \\
\hline $\begin{array}{l}\text { Erupciones } \\
\text { ectópica }\end{array}$ & & & & \\
\hline
\end{tabular}

Con relación a los factores de riesgos en las maloclusiones en la tabla 5 se observa la presencia de caries proximales en ambos sexo y las restauraciones deficiente. Mas en el sexo femenino

Al estudiar según tipo de maloclusion y retraso mental, el 46. 0 \% correspondió a la primera clase de Angle.

Entre las características clínicas preponderó la versión vestibular de los incisivos superiores, con $43,0 \%$, seguida de la adaquia, con $27,9 \%$.

\section{Discusión.}

Los principales problemas de salud bucal en estos niños son la caries dental, la enfermedad periodontal y las maloclusiones. Algunos autores coinciden en que la prevalencia y severidad de estos problemas son elevadas y ocasionan mayores trastornos que en niños normales. ${ }^{9}$ 
La elevada prevalencia de maloclusiones en el sexo masculino encontrada en estos niños, coincide con Chávez Taset en su Tesis para optar por el Título de Especialista Estomatología General Integral, a diferencia del estudio realizado por Milanés donde no existió diferencia de sexo y la edad que predomino fue la de 12 años . (Tesis para optar por el título de Especialista de Estomatología General Integral)

Los factores de riesgo que influyen principalmente en la aparición de estas enfermedades son la mala higiene bucal, la dieta cariogénica y los hábitos deformantes, todos ellos modificables con una buena educación sanitaria. ${ }^{9}$

Son numerosas las causas que pueden originar o acelerar las posiciones incorrectas de los dientes y sus relaciones inadecuadas con los maxilares, entre las que se encuentran los elementos totalmente externos, sobre los cuales se puede actuar y que han sido ampliamente estudiados y tratados por algunos autores, con énfasis en los hábitos bucales deformantes, pues al conocerlos se puede realizar una correcta prevención y atender al paciente de manera integral, como el ser biopsicosocial que es. ${ }^{10}$

Todos los hábitos tienen su origen en el sistema neuromuscular, como reflejos de la contracción muscular, de naturaleza compleja, los cuales se aprenden. Inicialmente es un acto voluntario o consciente, que se convierte en involuntario o inconsciente cuando se arraiga. ${ }^{10}$

Los procedimientos habituales de higiene bucal no logran el propósito de controlar la placa dental de prevenir la caries dental y la enfermedad periodontal, dado el papel determinante que tiene la misma en la causa de ambas afecciones, las que a su vez pueden causar la pérdida dental prematura, uno de los factores de riesgo en las maloclusiones, en las que también juegan un papel fundamental los hábitos deformantes. Aspectos que se reflejan en nuestro estudio 
Con respecto a los factores de riesgo para la salud bucal, los resultados obtenidos se corresponden con estudios similares de Abreu. ${ }^{9-11}$

\section{Conclusiones.}

En los niños con discapacidad mental la prevalencia y severidad de los problemas de salud bucal son muy elevadas. En la educación y desarrollo de estos niños el papel decisivo corresponde a los padres, a la familia, auxiliados por los educadores y el equipo de salud.

\section{Bibliografia.}

1- Tan Castañeda N, Rodríguez Calzadilla A. Correspondencia entre la formación académica del estomatólogo relacionado con pacientes especiales y la práctica estomatológica integral. 2002 Sep-Dic; 38(3).

2- Valdés Herrera MA. Pacientes discapacitados remitidos al servicio hospitalario que pueden ser tratados en la consulta ambulatoria convencional. Instituto Superior de Ciencias Médicas de La Habana; 2008.

3- Ravaglia C. Aspectos psico-clínicos para la atención odontológica de los pacientes con discapacidad. 2001.

4 Agenda Nacional para la igualdad en discapacidades. 2013-1017. Disponible en :www.planificacion.gob.ec/wp-content/.../Agenda-Nacional-para-Discapacidades.pdf

5- -Plaza Costa A, Silvestre Donat FJ. Odontología en pacientes especiales. Valencia: Universitat de València; 2007.

6- Bullón Fernández P, Machuca Portillo G. La atención odontológica en pacientes médicamente comprometidos. Madrid: Laboratorios Normon, SA; 2006.

7- Arias Herrera SR, Muñoz Fernández LM, Romero González CM, Espeso Nápoles N. Propuesta de comunicación en salud bucal para niños con retraso mental ligero, padres y educadores. Archivo Médico de Camagüey .2005; 9(6). 
8- Katia García Flores, Deysi Suárez Zafra, Karelia de la Huerta Flores. PanorÁmica de la atención estomatologica a pacientes discapacitados o especiales. Revista de Ciencias Médicas La Habana 2013; 19(2).

9- Muñoz Fernández Liset, Arias Herrera Sury, Romero González Carmen, Hidalgo García Carmen Rosa. Diagnóstico de salud bucal en niños con retraso mental ligero. AMC. 2005 Ago; 9(4): 2231.

10- Reyes Romagosa Daniel Enrique, Torres Pérez Ivette, Quesada Oliva Leticia Maria, Milá Fernández Maricela, Labrada Estrada Herminia Esther. Deforming oral habits in 5-11 years children. MEDISAN. 2014 Mayo; 18(5):606-612.

11- Abreu MHNG. Portadores de deficiencias: de incapaces a participantes co-asistido no promocäo da saude bucal. Rev Belo Horizonte. 1999:145. 